\title{
SOCIAL-PSYCHOPHYSIOLOGICAL COMPLIANCE AS A PREDICTOR OF FUTURE TEAM PERFORMANCE
}

\author{
Robert A. HENNING ${ }^{1)}$ and Kristopher T. KORBELAK ${ }^{1)}$ \\ ${ }^{1)}$ University of Connecticut, U.S.A.
}

\begin{abstract}
Social psychophysiological compliance (SPC) was tested as a predictor of future team performance of two-person teams performing a self-paced projective tracking task under laboratory conditions. Undergraduate students $(N=16$ teams, aged 17 to 23 yrs) worked in parallel using separate $\mathrm{X}-\mathrm{Y}$ joysticks to guide a virtual object through a complex path. One team member controlled the horizontal position of the object while the other controlled vertical. Unexpected changes in task control dynamics occurred at a randomly-selected point in the path: either horizontal and vertical (HV) control were swapped between team members, directional control was reversed, or both HV swap plus directional reversal occurred. Higher cardiac SPC (cross correlation, lag $=0$ ) scores predicted lower tracking error from path centerline $(p<.01)$ but did not predict collision severity between object and path wall. The results indicate that SPC has some potential for assessing a team's readiness to handle unexpected task demands in the immediate future.
\end{abstract}

Key words: social psychophysiological compliance, teamwork, cybernetics

Psychophysiological techniques can be used for objective and continuous monitoring of the human response to computer-mediated tasks but most studies employing these techniques have focused on individuals rather than teams. Furthermore, the reported empirical relationships are largely based on correlations between psychophysiological changes and performance measures collected over the same time period. The present study represents an attempt to develop a new approach, moving from individual to social psychophysiology, and from examining simultaneous events to testing if changes in social psychophysiology can be used to predict team performance in the immediate future.

This research is guided by the behavioral cybernetic model developed by K.U. Smith and associates (Smith \& Smith, 1987) which assumes that: (1) human behavior is controlled as a closed-loop or cybernetic process; (2) closed-loop behavioral links are established between sensory feedback from design factors in the performance environment; and (3) that sensory feedback control is mediated by motor behavior. This theoretical perspective differs from most present-day cognitive models that classify motor behavior as an output or end product of mental processes, that has no significant role in either controlling or organizing subsequent behavior.

Looking beyond the obvious role of motor behavior in feedback control of the environment, K.U. Smith's cybernetic theory highlighted the many roles of motor activity in behavior organization such as dynamic and continuous control of posture, fine

Correspondence concerning this article should be addressed to Robert Henning, Psychology Department, University of Connecticut, 406 Babbidge Road, Storrs, CT 06269-1020, U.S.A. (e-mail: Robert.Henning@, UConn.edu). 
manipulative movements including speech, and control over sensation and perception via motor-sensory control (Smith \& Smith, 1987). More important to the present research effort, however, is the cybernetic view that all motor behaviors have the ability to impact future behaviors through reciprocal effects on a wide range of internal bioenergetic processes and physiological states including respiration, heart rate, glucose metabolism and catecholamine levels (Smith, 1973). Not to be confused with general physiological arousal, the reciprocal effects of skilled behavior offer a far greater potential for specific and dynamic control over subsequent behavior (i.e., feedforward control) due to the endless variety, manner and extent to which motor activity can be articulated. While skilled behaviors provide a means for feedforward control of behavior, such behaviors are still dynamically adjusted and "fine tuned" based on feedback.

On a social level and in team work, each individual must control the sensory feedback generated not only by his/her own behavioral movements and functioning, but also the sensory feedback created by interacting with one or more team members. The study of interpersonal and group reciprocal sensory feedback and sensory feedback control relationships represents the focus of social cybernetics, directed towards delineating the closed-loop, behavioral-physiological manifestations and properties of social-behavioral interaction (Smith, 1972; Smith, Henning, \& Smith, 1994, 1995; Smith \& Smith, 1987).

Similar to the importance of motor control in self regulation of behavior, social cybernetic theory predicts that ongoing social behaviors serve a crucial organizing role for both present and future social behaviors because of the reciprocal effects of motor behavior on internal physiological states explained earlier. Three specific modes of social tracking have been identified; imitative, parallel-linked, or series-linked; which represent design factors that limit the specific nature and extent of mutual and yoked control. Specific differences in the nature and extent of psychophysiological changes are directly related to the tracking relationships in each mode of social tracking. Evidence for matched psychophysiological changes during parallel social interactions can be found in a number of studies (Boyd \& Di Mascio, 1954; Hatfield, Cacioppo, \& Rapson, 1994; Levenson \& Gottman, 1983; Levenson \& Ruef, 1992).

Based on earlier laboratory research on team control (Wegner \& Zeaman, 1956) and social cybernetics (Kao \& Smith, 1971, 1977; Sauter \& Smith, 1971), social psychophysiological compliance was examined during the parallel-linked mode of social tracking (Henning, Boucsein, \& Gil, 2001). Each team member could exert dynamic control over a simulated inertial object in a task that simulated teleoperation in microgravity (Smith, Henning, \& Li, 1998), and each team member received immediate visual feedback about how the system responded to these control inputs. Team members could view upcoming task demands and adjust the pace of the task accordingly. Social psychophysiological compliance (SPC) was defined as the extent to which changes in physiological states among the participants exhibited close correspondence or reflected mutual influence, quantified through the use of appropriate scoring of the time-series psychophysiological data collected from each social participant. Thus, a measure of SPC was used to indirectly assess the extent that team members establish mutual control over 
their coordinate social behaviors, yielding three scores of SPC based on each bivariate time series of either electrodermal activity, HR variability, or breathing pattern. SPC was scored using both weighted coherence through an adaptation (Bohrer, Henning, \& Marden, 1992) of an analysis method originally introduced to score two-factor cardiorespiratory coupling (Porges et al., 1980), and also cross correlation analysis at lag $=0$ was also performed. Each set of SPC scores was then tested separately as a predictor of team performance and team coordination.

SPC was found to be predictive of team performance, with the larger weighted coherence and positive cross correlation scores associated with improved performance. The weighted coherence statistic appeared more robust as an index of SPC, with task completion time and team RMS tracking error predicted by all three SPC measures for electrodermal activity, HR variability, and breathing. However, cross correlation of HR variability also predicted task completion time and team RMS tracking error, and these were the strongest predictive relationships in the data. The clustering of these predictive relationships, all in the expected direction where increased psychophysiological compliance was associated with improved performance, provides convergent support for a positive linkage between SPC and concurrent team performance. The results did not support a counter interpretation that psychophysiological compliance was due to matched joystick control actions. Subsequent analyses revealed positive linkages between SPC and team member ratings of performance and coordination (Gil \& Henning, 2000).

In the present study, the team tracking task is similar to the one used in Henning et al. (2001) and Glynn, Fekieta, and Henning (2001) but without the inertial properties of the controlled object. As a means to test for a possible predictive relationship between SPC and future team performance, task control dynamics were changed in an unpredictable manner during task trials so that teams were forced to regain control over the same basic task but with new input control characteristics of the joysticks. Manipulation of task control dynamics in this manner was expected to make tests of a possible predictive relationship between SPC and team performance in the immediate future more robust, since the specific nature of teamwork immediately following any change in task control dynamics would be inherently more challenging and complex. Only the cross correlation measure of heart rate was employed since this was the leading indicator of SPC in Henning et al. (2001), and because it is more amenable to calculation over the short portions of the trial periods than the weighted coherence scoring technique.

\section{METHOD}

\section{Participants:}

As part of a larger study on the use of force feedback to promote teamwork, 32 undergraduate psychology students working in two-person teams $(2 \mathrm{MM}, 8 \mathrm{FF}$, and $6 \mathrm{MF}$ teams; mean aged=18.8 yrs, $S D=1.2$ yrs) received course credit for participating.

\section{Team Task:}

Team members sat side-by-side facing a $81.3 \mathrm{~cm}$ diagonal television monitor approximately $76 \mathrm{~cm}$ away that displayed a simulated square object within a 2-dimensional complex path (see Fig. 1). Each team 


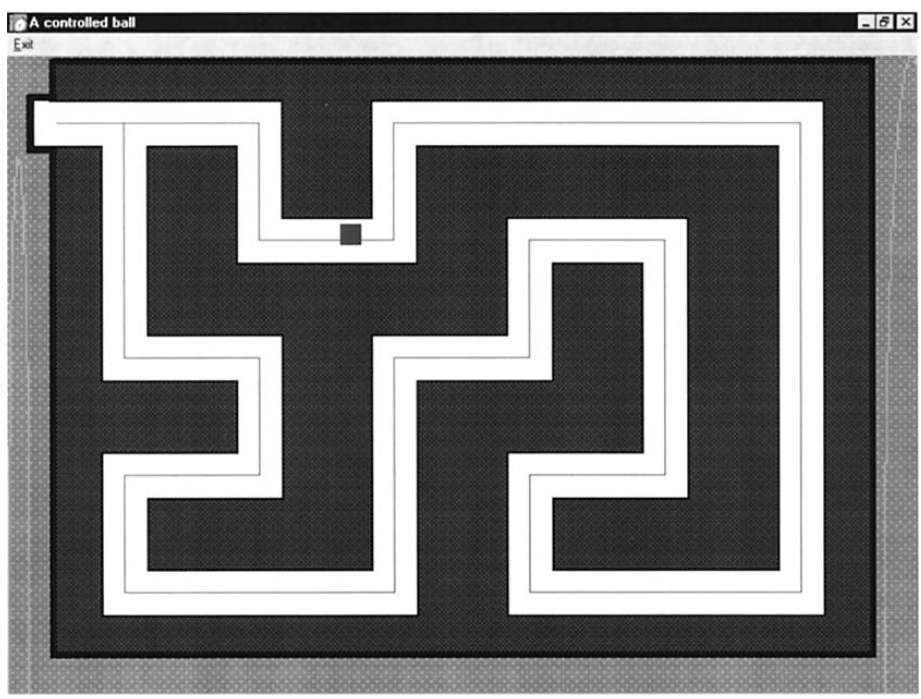

Fig. 1. Complex path showing simulated object and path centerline.

member operated a computer-interfaced force feedback joystick (Impulse Engine ${ }^{\mathrm{TM}}$ 2000, Immersion Corporation, San Jose, CA) to control either the vertical or horizontal movement of the square object in the complex path. The system sample rate was $50 \mathrm{~Hz}$. Navigating the square object through the path from start to finish required team coordination of both horizontal and vertical joystick inputs. The computer-interfaced joysticks were used to simulate centering spring forces typical of most joysticks, or to deliver coordinate forces proportional to the discrepancies between the horizontal and vertical joystick inputs of both team members. In this latter case, the joystick forces provided a source of dynamic relational feedback indicating how well the team members were tracking (i.e., matching) each other's X-Y joystick control inputs. A divider prevented either team member from viewing the other team member's joystick movements.

\section{Experimental Design:}

A within-subjects (within-teams) experimental design was used to control for potential differences between teams. Practice trials both with and without relational force feedback prior to experimental conditions minimized learning effects. In most experiment trials, changes in task control dynamics, in which horizontal or vertical control was swapped unexpectedly between team members, occurred somewhere between $25 \%$ and $75 \%$ of the path length (see Fig. 1). Three types of changes in task control dynamics occurred throughout the experiment: 1) horizontal and vertical (HV) control were swapped between team members, 2) directional control was reversed (i.e., a participant's control might change from compliant horizontal to non-compliant [reversed] horizontal such that moving the joystick to the left would move the object to the right), or a combination of both HV swap plus directional reversal. In a few experimental trials, task control dynamics remained unchanged throughout the trial to make the task changes less predictable.

Every team performed 2 practice trials and 24 experimental trials. Changes in task control dynamics within the first 12 experimental trials were identical to changes in task control dynamics within the last 12 experimental trials. Specific changes in task control dynamics were initially randomly selected from a matrix containing all possible changes for this task. Trial conditions were then coupled so that participants began each trial with the same type of task control dynamics they had at the conclusion of the previous trial, thus eliminating the need to learn new task control dynamics at the beginning of each new trial. Exposure to relational force feedback was counterbalanced across teams to control for order effects, with one block of 12 trials providing relational force feedback, and the other block providing the more familiar centering spring forces common to most joysticks. 


\section{Measures:}

Task Performance: RMS tracking error and collision severity. RMS tracking error was measured as the root-mean-squared distance error between the center of the square object and the centerline of the complex path. Collision severity was scored as the number of contact collisions the simulated object had with path walls, multiplied by the average position error that would have occurred if the object had not been stopped by the path wall (i.e. had the object been allowed to continue moving through the path wall in response to erroneous joystick control inputs). Separate scores were calculated before and after the point in time when task control dynamics changed, and also for the $3.5 \mathrm{~s}$ immediately after the change in task control dynamics.

Social Psychophysiological Compliance (SPC). Each team member's electrocardiogram was monitored using disposable surface electrodes and a telemetry system (UFI Corp.). An R-wave detector (CWE Corp.) and a period meter enabled interbeat intervals to be measured within $1 \mathrm{msec}$. The interbeat interval values were sampled at $5 \mathrm{~Hz}$ to create a time series for each team member that was later used for cross correlation analysis (at lag $=0$ ) to score the degree to which the team members' changes in heart rate reflected social psychophysiological compliance. Interbeat interval values had to be greater than $330 \mathrm{msec}$ and less than $2000 \mathrm{msec}$ for both team members simultaneously, and for more than half of each scoring period in order for the scores to be considered valid for statistical analyses. Separate SPC scores were calculated before and after the point in time when task control dynamics unexpectedly changed, and also for the $3.5 \mathrm{~s}$ periods both immediately before and immediately after the change in task control dynamics.

\section{Procedure:}

After obtaining informed consent, participants were instructed on how and where to place the cardiac surface electrodes on their skin in private. Team members were then seated in front of the video monitor and received an explanation and demonstration of the task and the proper use of the joysticks. Participants were instructed to quickly and successfully navigate the square object through a randomly selected complex path, while minimizing distance from the centerline in the complex path and also avoiding collisions with the path walls (see Fig. 1). Participants were allowed to talk to each other during the experimental trials if they requested this. Next, teams completed a practice trial, with control dynamics identical to the first experimental trial except that no change in task control dynamics occurred during this practice trial. Teams then completed the 12 experimental trials that included centering spring force feedback (or relational force feedback). A second practice trial occurred prior to the second block of 12 trials that included relational force feedback (or centering spring force feedback).

Analysis:

A data screening approach required interbeat intervals to be valid $(330 \mathrm{msec}<\mathrm{IBI}<2000 \mathrm{msec})$ simultaneously for both team members for more than half of each scoring period. A simple correlation analysis was performed to determine if the psychophysiological synchronization scores prior to a change in task control dynamics were associated with either RMS tracking error or collision severity following the mid-trial change in task control dynamics. In addition, a set of targeted, separate multiple regression analyses were performed to examine more closely the predictive relationships between SPC and future team performance. These regression analyses included dummy predictor variables to help isolate the effects of changes in SPC on within-team changes in performance. Dummy predictor variables were included for each team and also for the presence or absence of relational force feedback.

\section{RESULTS}

Trial completion times averaged about $18 \mathrm{~s}(\min =5.4 \mathrm{~s}, \max =60.68 \mathrm{~s})$. Data screening for acceptable cardiac IBI values, plus the requirement that the IBI values from each team member were simultaneously valid, yielded 275 valid trials from 16 teams. Descriptive statistics are found in Table 1. Based on the descriptive data in Table 1, RMS tracking performance tended to improve across each complete trial, and performance in the $3.5 \mathrm{~s}$ 
Table 1. Descriptive Statistics

\begin{tabular}{|c|c|c|c|c|c|c|}
\hline & \multicolumn{2}{|c|}{$\mathrm{SPC}^{*}$} & \multicolumn{2}{|c|}{ RMS Error } & \multicolumn{2}{|c|}{ Collision Severity } \\
\hline & Mean & $S D$ & Mean & $S D$ & Mean & $S D$ \\
\hline $3.5 \mathrm{~s}$ Pre-Change & .53 & .52 & - & - & - & - \\
\hline Pre-Change & .63 & .38 & 37.5 & 8.1 & 1282 & 1717 \\
\hline 3.5 s Post-Change & .56 & .48 & 35.1 & 5.6 & 14974 & 13268 \\
\hline Post-Change & .53 & .43 & 33.9 & 5.0 & 3049 & 2224 \\
\hline Overall & .57 & .35 & 35.3 & 5.3 & 41943 & 46553 \\
\hline
\end{tabular}

Note: $\mathrm{SPC}=$ Social Psychophysiological Compliance.

Table 2. Regression Analyses for SPC and RMS Tracking Error

\begin{tabular}{lccc}
\hline \multicolumn{1}{c}{ Predictor } & Pre-Change & 3.5 s Post-Change & Post-Change \\
\hline SPC 3.5 s Pre-Change & - & -.183 & - \\
SPC Pre-Change & .053 & - & $-.209^{\dagger}$ \\
SPC 3.5 s Post-Change & - & $.200^{*}$ & - \\
SPC Post-Change & - & - & -.026 \\
\hline
\end{tabular}

Notes: Table consists of Beta weights, ${ }^{\dagger} p<.10$ (trend). ${ }^{*} p<.05$.

$\mathrm{SPC}=$ Social Psychophysiological Compliance.

Table 3. Regression Analyses for SPC and Collision Severity

\begin{tabular}{lccc}
\hline \multicolumn{1}{c}{ Predictor } & Pre-Change & 3.5 s Post-Change & Post-Change \\
\hline SPC Pre-Change & $-.260^{*}$ & -.041 & -.031 \\
SPC 3.5 s Post-Change & - & - & - \\
SPC Post-Change & - & - & -.090 \\
\hline
\end{tabular}

Notes: Table consists of Beta weights, ${ }^{*} p<.05$.

$\mathrm{SPC}=$ Social Psychophysiological Compliance.

period following the unexpected change in task control dynamics is shown to be temporarily degraded, reflecting increased task challenge as intended.

The simple correlation analysis (no covariate adjustments) revealed pre-change SPC negatively predicted post-change $R M S$ tracking error $(t=-3.45, p<.001)$, explaining 3.8 percent of performance variance across all teams and conditions. SPC did not similarly predict collision severity ( $p>.05)$. A summary of the targeted multiple regression outcomes is provided in Tables 2 and 3. Only limited support was found, with pre-change SPC predicting post-change $R M S$ tracking error at the level of a trend $(p<.10)$, but showing the same directional relationship as the simple correlation, namely pre-change SPC negatively predicting post-change RMS tracking error. 


\section{DISCUSSION}

This small-sample laboratory study was able to provide some limited empirical evidence that social-psychophysiological compliance (SPC) can be used to predict team performance in the immediate future. In the computer-based team task that required coordination of horizontal and vertical joystick inputs by two team members, increased levels of SPC in the task period prior to a change in task control dynamics was associated with lower team tracking error in the post-change period, thus indicating that SPC can be used to predict team performance in the immediate future. The finding that SPC could predict team social tracking performance after this challenging change in task control dynamics suggests that this predictive relationship is relatively robust. SPC was not predictive of collision performance, and this can be explained by the nature of collisions that are sporadic and hard to quantify versus RMS tracking error which can be assessed continuously.

The presence of a trend rather than statistical significance in the more targeted multiple regression analysis of RMS tracking error may be a consequence of the extent of missing data. The electrode-based amplifier system (Fetrode ${ }^{\mathrm{TM}}$, UFI Corp.) used in the present study had eliminated most of the artifacts caused by electrode lead movements but artifacts due to EMG activity during active joystick control still remained. In future studies, this problem could be addressed through incorporation of statistical estimation procedures for missing data.

The possibility that SPC might predict team performance in the immediate future has a theoretical basis in behavioral cybernetics (Smith \& Smith, 1987). Coordinate motorsensory control is considered fundamental to dynamic social tracking activities, and promotes social psychophysiological compliance that can later facilitate coordination of future motorsensory control inherent to teamwork. Thus the presence of SPC would not only benefit ongoing motorsensory control during social tracking. The reciprocal effects of SPC on social-behavioral organization and control would also increase social proficiency and benefit team performance in the immediate future.

The specific time period that SPC can benefit future teamwork behaviors is not yet clear, and is in need of study. The length of trial periods in the present study suggests that the beneficial effects of SPC lasted for at least a portion of the post-change periods (about $10 \mathrm{~s}$ ). One can speculate that the time course over which the reciprocal effects of SPC benefit teamwork is likely to exponentially decay when the originating coordinate motorsensory behaviors come to an abrupt end. If this is so, a better way to characterize the influence of SPC would be in terms of a half-life period. However, there is also the possibility that the half-life period of SPC may differ depending on the mode of social tracking involved and also the feedback control dynamics of the task environment. For example, the benefits of SPC in hormonal changes may have a long half-life period lasting minutes or hours in social relationships, while the benefits of SPC linked to cardiac changes during simple tasks like the one in the present study may have a half-life period only lasting several seconds. SPC with an intermediate half-life period may be more beneficial to teams engaged in joint decision making since tasks of a cognitive nature 
require time-consuming transformations of sensory feedback for effective coordinate control of cognition.

One possible application of the present findings is in the area of team biocybernetics, where SPC could be used to gauge teamwork capabilities in the immediate future. Task challenge levels and/or the extent of task automation could be dynamically adjusted on a proactive basis using SPC, thus avoiding conditions of task demand that no longer match the team's capabilities. This could also help solve the practical problem of time delays inherent to complex sociotechnical systems, whereby adjustments based on SPC monitoring could make the overall system respond sooner. A team biocybernetic system operating on these principles can be considered to have rudimentary social tracking skills reflected in its ability to track and predict the capabilities of the human team, and perhaps this level of social skill is necessary before human teams can work with a high level of proficiency in conjunction with complex automated systems.

\section{REFERENCES}

Bohrer, R., Henning, R. A., \& Marden, J. I. 1992. A measure of synchronicity in multiple time series analysis. Unpublished manuscript, University of Connecticut, Storrs, CT.

Boyd, R. W., \& Di Mascio, A. 1954. Social behavior and autonomic physiology (a socio-physiologic study). Journal of Nervous and Mental Disease, 120, 207-218.

Gil, M. C., \& Henning, R. A. 2000. Determinants of perceived teamwork: examination of team performance and social psychophysiology. Proceeding Triennial Congress of the Int. Ergonomics Association and 44th Annual Meeting of the Human Factors and Ergonomics Society, 2, 743-746.

Glynn, S. J., Fekieta, R., \& Henning, R. A. 2001. Use of force-feedback joysticks to promote teamwork in virtual teleoperation. Proceedings of the 45th Annual Meeting of the Human Factors and Ergonomics Society, October 8-12, Minneapolis, MN.

Hatfield, E., Cacioppo, J. T., \& Rapson, R. L. 1994. Emotional Contagion. Cambridge University Press.

Henning, R. A., Boucsein, W., \& Gil, M. O. 2001. Social-physiological compliance as a determinant of team performance. International Journal of Psychophysiology, 40, 221-232.

Kao, H. S., \& Smith, K. U. 1971. Social feedback: determination of social learning. Journal of Nervous and Mental Disease, 152, 289-297.

Kao, H. S., \& Smith, K. U. 1977. Delayed visual feedback in inter-operator social tracking performance. Psychologia, 20, 20-27.

Levenson, R. W., \& Gottman, J. M. 1983. Marital interaction: Physiological linkage and affective exchange. Journal of Personality and Social Psychology, 45, 587-597.

Levenson, R. W., \& Ruef, A. M. 1992. Empathy: A physiological substrate. Journal of Personality and Social Psychology, 63, 234-246.

Porges, S. W., Bohrer, R. E., Cheung, M. N., Drasgow, F., McCabe, P. M., \& Keren, G. 1980. New time series statistic for detecting rhythmic co-occurrence in the frequency domain: the weighted coherence and its applications to psychophysiological research. Psychological Bulletin, 88, 580-587.

Sauter, S. L., \& Smith, K. U. 1971. Social feedback: quantitative division of labor in social interactions. Journal of Cybernetics, 1, 80-93.

Smith, K. U. 1972. Cybernetic psychology. In R. N. Singer (Ed.), The psychomotor domain (pp. 285-348). New York: Lea and Febiger.

Smith, K. U. 1973. Physiological and sensory feedback of the motor system: Neural metabolic integration for energy regulation in behavior. In J. Maser (Ed.), Efferent organization and the integration of behavior (pp. 19-66). New York: Academic Press.

Smith, T. J., Henning, R. A., \& Li, Q. 1998. Teleoperation in Space - Modeling Effects of Displaced Feedback and Microgravity on Tracking Performance. SAE Technical Report \#981701. 28th 
International Conference on Environmental Systems, Danvers, MA, July 13-16.

Smith, T. J., Henning, R. A., \& Smith, K. U. 1994. Sources of performance variability. In G. Salvendy \& W. Karwowski (Eds.), Design of work and development of personnel in advanced manufacturing (Chap. 11, pp. 273-330). New York: Wiley.

Smith, T. J., Henning, R. A., \& Smith, K. U. 1995. Performance of hybrid automated systems - a social cybernetic analysis. International Journal of Human Factors in Manufacturing, 5, 29-51.

Smith, T. J., \& Smith, K. U. 1987. Feedback-control mechanisms of human behavior. In G. Salvendy (Ed.), Handbook of human factors (pp. 251-293). New York: Wiley.

Wegner, N., \& Zeaman, D. 1956. Team and individual performances on a motor learning task. The Journal of General Psychology, 55, 127-142.

(Manuscript received January 22, 2005; Revision accepted April 26, 2005) 